\title{
Uncertainty in pigeons
}

\author{
LESLIE M. SOLE, SARA J. SHETTLEWORTH, and PATRICK J. BENNETT \\ University of Toronto, Toronto, Ontario, Canada
}

\begin{abstract}
Pigeons classifieda display of illuminated pixels on a touchscreen as sparse or dense. Correct responses were reinforced with six food pellets; incorrect responses were unreinforced. On some trials an uncertain response option was available. Pecking it was always reinforced with an intermediate number of pellets. Like monkeys and people in related experiments, the birds chose the uncertain response most often when the stimulus presented was difficult to classify correctly, but in other respects their behavior was not functionally similar to human behavior based on conscious uncertainty or to the behavior of monkeys in comparable experiments. Our data were well described by a signal detection model that assumed that the birds were maximizing perceived reward in a consistent way across all the experimental conditions.
\end{abstract}

Researchers are increasingly interested in studying higher cognitive processes and even consciousness in nonhuman animals (e.g., Clark \& Squire, 1998; Griffin, 2001; Macphail, 1998; Smith, Shields, Schull, \& Washburn, 1997; for a review, see Shettleworth, 1998). Although it is not possible to know whether animals' behavior is accompanied by human-like subjective experiences, it is possible to design nonverbal tests for processes that people normally evidence by verbal report and look for behavior that is functionally similar to human behavior (see Hampton, 2001; Shettleworth, 2001). To take the example of interest in this article, metacognition has been studied by asking people what they think they know, perceive, or remember while concurrently testing their knowledge and so forth to see whether those self-reports are accurate (Nelson, 1996). If people are able accurately to report on their own cognition, they are considered to have metacognition. By analogy, to test a nonverbal creature for a metacognitive process, it is necessary to have both a way of asking the animal "How well do you think you're going to perform?" and a direct measure of that performance. One way to do this is to provide the animal with an escape from the test of cognition. Choice of this option is rewarded, but not as well as correct performance on the primary task. Subjects that know on a trial-by-trial basis how well they will perform on the primary task should selectively escape from difficult trials when allowed to. Moreover, when they choose to complete the primary task instead of opting out, they should perform better on average than when forced to complete it.

This article is based on a B.Sc. thesis by the first author. Some of the data were reported at the Conference on Comparative Cognition, Melbourne, FL, March 1998. The research was supported by research grants from the Natural Sciences and Engineering Research Council of Canada to the second and third authors. We thank Rick Westwood and David Kabelik for technical assistance and Robert Hampton for comments on various versions of the manuscript. Correspondence may be directed to S. J. Shettleworth, Department of Psychology, University of Toronto, 100 St. George Street, Toronto, ON, M5S 3G3 Canada (e-mail: shettle@ psych.utoronto.ca).
In several recent studies, researchers have taken the approach sketched above to test various nonhuman animals for aspects of metacognition. Smith and his colleagues (Shields, Smith, \& Washburn, 1997; Smith et al., 1995; Smith et al., 1997) reported that monkeys and a dolphin perform similarly to people on a nonverbal test of perceptual uncertainty. In one study, rhesus monkeys and people classified the density of illuminated pixels on a video screen as high or low (Smith et al., 1997). The high density was constant throughout the experiment, but the low density could be anything from very low up to a density almost indiscriminable from high. The subjects were allowed to escape from classifying the displays by choosing an uncertain response, which led, after a short delay, to a rewarded trial with an easy stimulus. So that the uncertain response was not used too often, the delay imposed between it and the next, easy trial was increased as a function of its recent frequency. The humans and the monkeys escaped most often from trials in which the stimulus was near the threshold of discriminability; performance without the escape option was not systematically assessed. Similarly, in an auditory discrimination in which tones of exactly $2100 \mathrm{~Hz}$ were to be classified high and tones between 1200 and $2099 \mathrm{~Hz}$ were to be classified low, people and a bottlenosed dolphin escaped most often from trials with near-threshold stimuli (Smith et al., 1995). Human subjects in both of these studies said that they responded high or low to certain stimuli but escaped when they were uncertain. The similar pattern of data from the monkeys, the dolphin, and the people is consistent with the possibility that their behavior was accompanied by similar subjective states but need not compel this conclusion.

All the data in the studies of Smith et al. (1995; Smith et al., 1997) were described by a signal detection model in which two decision criteria divide the stimulus continuum into three regions corresponding to low, uncertain, and high. However, applications of signal detection theory do not necessarily imply that subjects are consciously setting criteria and weighing the costs and benefits of different 
courses of action (Macmillan \& Creelman, 1991). Indeed, such models provide an excellent fit to many data from pigeons, animals less readily thought of than monkeys and dolphins as engaging in such activities (D. S. Blough, 1967; Boneau \& Cole, 1967; Commons, Nevin, \& Davison, 1991; Marston, 1996; Shettleworth, 1998; Wixted, 1993). A reinforcement-based model without explicit stimulus representations and decision criteria also accounts for many such data (White \& Wixted, 1999). These approaches to modeling the data are not incompatible with accounts in terms of subjective states, but neither do they compel such accounts.

Metacognitive uncertainty has been studied in pigeons by Inman and Shettleworth (1999), who looked for evidence of metamemory in a three-alternative delayed matchingto-sample task. Correct matching was reinforced with six food pellets, and incorrect responses were unreinforced. On some trials, the pigeons were also given a safe key, analogous to the uncertain response in the studies by Smith and colleagues. Pecking the safe key led immediately to the delivery of three food pellets, less than the six pellets obtainable by correct matching but more than could be obtained by chance performance on the three-alternative matching task. Opportunities to choose between sample and distractors and the safe key were intermixed with matching trials without the safe key and with trials ending in the safe key alone. All the birds pecked the safe key most often at the longest retention intervals, when memory was worst, and they matched more accurately when they freely chose among the sample and the distractorsthat is, with the safe key available - than when forced to take the test of memory. This pattern of behavior is consistent with the pigeons' being able to assess their own memory strength. However, they no longer performed in a way consistent with metamemory on a more stringent test in which they were forced to choose between access to the safe key and a test of memory at the end of the retention interval, before the sample and the distractors appeared. Here, the birds performed no differently on forced and freely chosen memory tests. In contrast, rhesus monkeys given the same kind of test, as well as novel transfer tests, performed as if they knew whether or not they remembered the sample (Hampton, 2001).

Comparing the findings of Inman and Shettleworth (1999) with those of Hampton (2001; see also Smith, Shields, Allendoerfer, \& Washburn, 1998) suggests there may be species differences in metamemory between monkeys and pigeons. The present experiment extends the investigation of functional similarities among species in metacognitive tasks by testing pigeons' perceptual uncertainty in a difficult visual discrimination. Like Smith et al. (1997) in their studies of monkeys, we trained our animals to classify video images as having a low or a high density of illuminated pixels. Unlike Smith et al. (1997), we used only one near-threshold pixel density, chosen in preliminary testing to be difficult for the birds to discriminate from the high density. Testing whether or not animals show evidence of metacognition does not depend on hav- ing multiple near-threshold stimuli. What is important is to rule out the possibility that rather than assessing their own cognition, subjects simply learn that, for specific stimuli, escaping received greater average reward than did performing the primary discrimination task. An animal that is sensitive, on a trial-by-trial basis, to its memory strength or perceptual certainty should most often choose to complete the task on trials in which it will perform accurately. In the present experiment, as in Inman and Shettleworth (1999, Experiment 1), we tested whether pigeons could do this by randomly mixing trials with and without the safe key. In contrast, most of the experiments by Smith and colleagues tested performance without the escape option only occasionally. This makes it difficult to tell whether the subjects performed less accurately on the primary discrimination when forced to complete it than when the uncertain response was available but rejected, when the subjects presumably were certain they could perform well. Also unlike Smith and colleagues, we provided a safe key with a fixed but mediocre reward, rather than one that diminished in value when it was chosen too often. This facilitated optimality analyses of the birds' performances, as well as increasing the likelihood that they knew the payoff for pecking the safe key. To increase that likelihood and to counterbalance the trials in which it was absent, the safe key occasionally appeared alone after the display of pixels, and the birds had no choice but to peck it and receive its associated reinforcer. Originally, that reinforcer was four food pellets, less than the six-pellet payoff for correct classification of the pixel displays but greater than that obtainable for chance performance on the classification task. However, like other animals (see Kacelnik \& Bateson, 1996), pigeons tend to prefer a constant amount of food to a variable amount with the same mean (Hamm \& Shettleworth, 1987). That is, they are risk averse in reward amounts. Not surprisingly, therefore, a constant three pellets was sufficient to maintain fairly frequent choice of the safe key by 2 of our birds.

Just as a person can report conscious uncertainty about classifying any of a variety of stimuli, an animal that chooses a safe option when it is uncertain should continue to do so when perceptual uncertainty is produced in a new way. Transfer tests of this kind can help to distinguish responding based on metacognition from responding in specific ways to specific external stimuli. Hampton (2001) and Shields et al. (1997) included transfer tests in their studies of monkey metamemory, with positive results, but previous studies of perceptual uncertainty (Shields et al., 1997; Smith et al., 1995) have not systematically tested transfer. Here, while being trained and tested with green stimuli, the birds were occasionally exposed to high or low densities of red pixels and were reinforced for classifying them correctly. We assumed that the birds would treat the red and the green stimuli as different, because color is generally a very salient dimension for pigeons. An intermediate density of red pixels was displayed for the first time in the transfer phase to test whether the birds would transfer use of the safe key to a novel near-threshold stim- 
ulus. Continued testing with the red stimuli provided a second set of data on stable performance.

In summary, in this experiment, we investigated use of an uncertain response by pigeons in a difficult visual discrimination task. We asked three questions. First, will pigeons use this response most frequently on trials in which the stimulus is most difficult to classify? Second, can the data be described by a simple signal detection model that assumes that the birds will respond in a way that maximizes perceived reward? Third, is the uncertain response specific to the stimuli used in training, or does it generalize to novel near-threshold stimuli? If the uncertain response is functionally similar to human reports of uncertainty, the birds should use it selectively with the most difficult stimuli, transfer it to a new near-threshold stimulus, and make fewer discrimination errors on freely chosen than on forced discrimination tests.

\section{METHOD}

\section{Subjects}

The subjects were three 2- to 5-year-old White King pigeons (Columba livia) with extensive histories of visual discrimination training, maintained at $85 \% \pm 2 \%$ of their free-feeding weights. Between their last experience in an experiment and this one, they had lived socially in a large aviary with free food and water.

\section{Apparatus \\ Each pigeon was tested in a separate $40.5 \mathrm{~cm}$ high $\times 30.0 \mathrm{~cm}$ wide $\times 29.0 \mathrm{~cm}$ long Plexiglas chamber with one wall opening onto a $19.0 \mathrm{~cm}$ high $\times 26.5 \mathrm{~cm}$ wide flat screen video monitor. The screen was covered with a thin sheet of nonreflecting Plexiglas and was crisscrossed by a grid of infrared beams that detected pecks directed at the monitor (touchframe; Carrol Touch, Round Rock, TX). A food tray below the screen caught 20 -mg food pellets delivered from out- side the chamber.}

\section{Procedure}

Following preliminary training, ${ }^{1}$ each trial began with illumination of a specified proportion of red or green pixels in a $3 \times 3 \mathrm{~cm}$ square centered halfway across the monitor and $12.5 \mathrm{~cm}$ above the base. The screen was otherwise dark. The green dense (GD) and red dense (RD) stimuli had a random $80 \%$ of the pixels illuminated; the green sparse (GS) and red sparse (RS) stimuli had 20\%. In the stages for which data are reported, the green variable $(\mathrm{GV})$ and red variable (RV) stimuli had pixel densities of $70 \%$. The first peck anywhere on this sample stimulus began a 4-sec observing interval, at the end of which the next peck turned off the sample and turned on one or more of three possible stimuli at the side(s) of the screen. Each of these measured $2.3 \mathrm{~cm}$ at its widest point. On matching trials, a yellow star and a blue triangle were centered $8.5 \mathrm{~cm}$ from one edge of the screen, with the higher shape $2.5 \mathrm{~cm}$ below the top of the screen and the other centered $9.6 \mathrm{~cm}$ below it. The side on which they appeared and which one was above the other varied randomly. After GD or RD samples, one peck to the star was reinforced with six pellets. One peck to the triangle was reinforced with six pellets after any sample of less than $80 \%$ density (GS, GV, RS, or RV). Incorrect responses led immediately to a 6-sec time out (the same as the reinforcement period) and the 20 -sec intertrial interval. The safe key was a purple circle centered $8.5 \mathrm{~cm}$ from one side of the touchscreen and $12.2 \mathrm{~cm}$ above the bottom. On safe key trials, the safe key appeared alone, randomly on the left or right side of the screen. All three choice stimuli appeared on combined trials, with the safe key on one side of the screen and the star and triangle on the other. In the first phase of testing, sessions consisted of 80 combined, 40 matching, and 40 safe key trials mixed randomly. GD, GS, GV, RD, and RS samples were intermixed in each trial type in the ratio 9:3:6:1:1.

Data were analyzed in 4-session blocks. Choice of the safe key was reinforced with four pellets initially, but this was reduced to three pellets for Bird 57 after one block and for Bird 80 after two blocks because they chose the safe key on most combined trials. All birds completed four blocks of sessions with their final values of safe key reward; the data from the final three blocks are presented here as representing stable performance. Transfer testing with RV samples began immediately thereafter and lasted for 16 sessions. RD, RS, RV, GD, and GS samples were intermixed in the ratio 9:3:6:1:1 within matching, safe-key, and combined trials, which were conducted as before. Data from the last three blocks of 4 sessions were taken as representing stable performance with these stimuli.

\section{Results and Discussion}

As was planned, the birds classified the near-threshold GV and RV stimuli less accurately than the sparse and dense stimuli both on matching-only trials, when they were forced to classify the stimuli, and on combined trials, when the safe key was available. (Percentage of correct matching on combined trials is the number of correct choices of the star or triangle as a proportion of combined trials on which the bird pecked one of these shapes rather than the safe key.) Like the dolphin, monkeys, and people tested by Smith et al. (1995; Smith et al., 1997), the pigeons often chose the safe option on these most difficult trials. However, they performed no better when choosing to classify the stimulus on combined trials than when forced to classify it on matchingonly trials. In initial testing, the effect of stimulus on proportion correct was highly significant $[F(4,8)=10.75$, $p<.01$; for trial type, $F(1,2)=2.85$; for stimulus $\times$ trial type, $F(4,8)=0.528$ ]. The pattern of results was similar for the last three blocks of the transfer phase [for stimulus, $F(4,8)=8.12, p<.01$; for trial type, $F(1,2)=0.391$; and for stimulus $\times$ trial type, $F(4,8)=1.18$ ]. Fisher's least significant difference (LSD) comparisons showed that accuracy on the GV stimulus was significantly lower than accuracy on the other four stimuli. However, although in nearly all cases the birds classified the dense stimuli less accurately than the sparse ones (see Figure 1), the LSD post hoc test did not show these differences to be significant.

Choices of the safe key also differed significantly across stimuli in both phases [in original training, $F(4,8)=8.06$, $p<.01$; in transfer, $F(4,8)=5.64, p<.05]$. Although the birds classified GD samples more accurately than GV samples, they did not respond differently to the safe key when these samples were presented. Fisher LSD multiple comparisons showed that, in training, safe key choices following GS, RS, and RD samples differed from those following GD or GV samples, but these two subsets of data did not differ. Similarly, during transfer testing, safe choices following RD and RV did not differ.

What response strategy maximizes the average reward when the safe key is present? The answer depends on the perceived values of the rewards given for correct, incorrect, and safe key responses. The simplest case is one in 


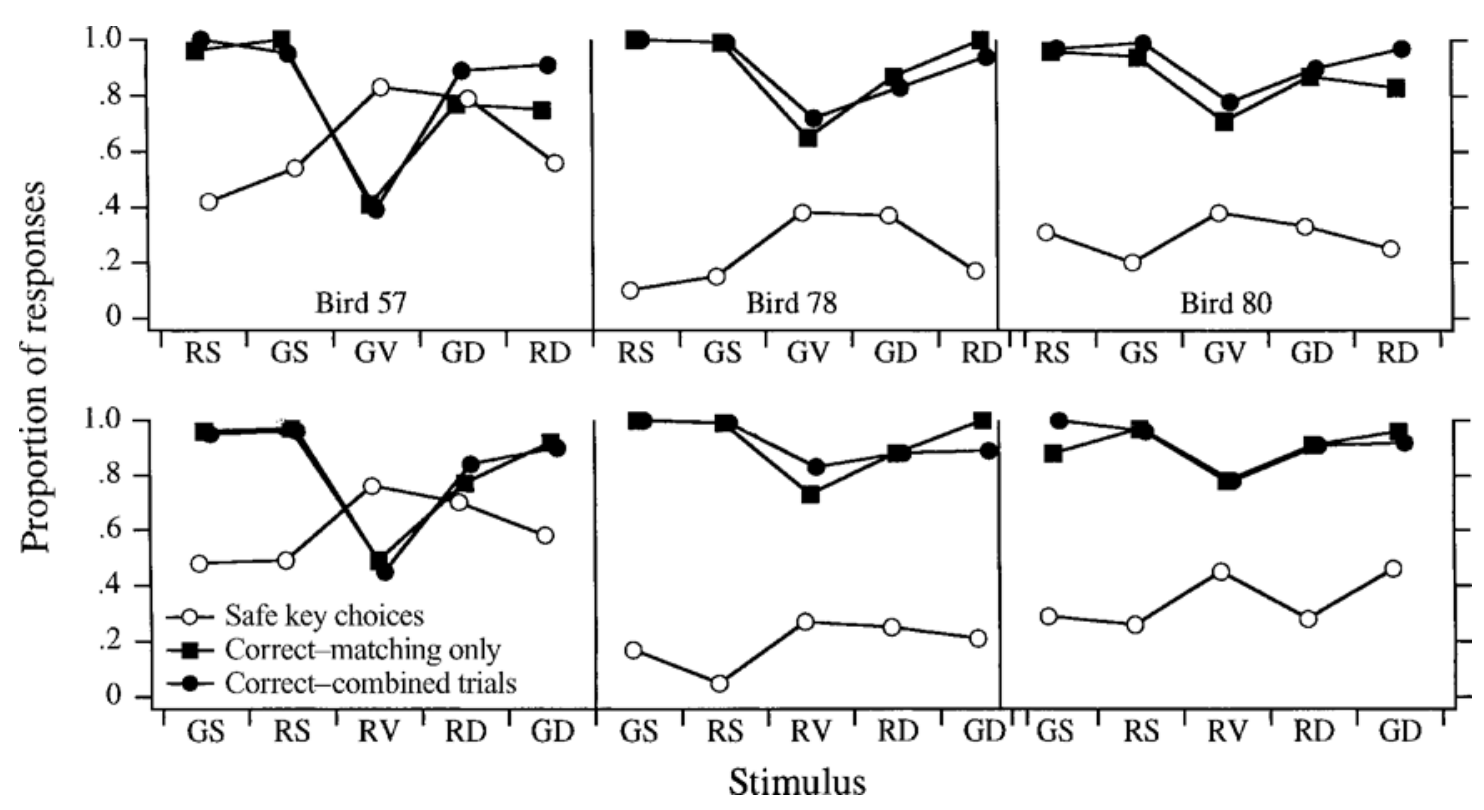

Figure 1. Proportions of correct classification responses on matching-only and combined trials and proportion of combined trials with a choice of the safe key for the 3 birds in the last three blocks of four sessions in the original test (upper row) and transfer (bottom row) phases. Proportion correct on combined trials is based on trials in which the birds did not choose the safe key. G, green; R, red; S, sparse; D, dense; V, variable.

which perceived value is proportional to the number of food pellets. In this case, the strategy that maximized the average reward would be identical to the single-criterion strategy used on trials when the safe key was absent. ${ }^{2}$ Such is not the case when the value of the reward for selecting the safe key is increased relative to the reward for a correct response. In the extreme case in which correct and safe key rewards are valued equally, selecting the safe key on every trial would maximize the average reward. For intermediate cases, the ideal strategy is to compare the internal response to two criteria that fall above and below the optimal one: The bird should respond dense whenever the internal response exceeds the relatively conservative criterion, respond sparse whenever it is less than the relatively lax one, and respond safe whenever it falls between the two criteria (see Figure 2).

We compared the data from the combined trials to the predictions of a signal detection model that assumed (1) that the average perceived reward for a correct matching response (i.e., correctly selecting sparse or dense) was $w$ times the perceived value of the reward for selecting the safe key, (2) that $w$ was the same value for all the birds and all the conditions, (3) that the reward for an incorrect matching response was zero, and (4) that the decision criteria were set to maximize the average perceived reward. For each bird, standard equations (Macmillan \& Creelman, 1991) were used to convert the proportion of correct responses to each stimulus to two $d^{\prime}$ values and decision criteria (see Table 1). The $d^{\prime}$ values represented the differences between the means of the sparse, variable, and dense internal distributions. The two criteria divided the decision axis into three regions: The model responded sparse whenever the internal response was less than both criteria, dense when the response was greater than both criteria, and safe when the response was in-between the criteria (see Figure 2). MATLAB (The Mathworks) was used to calculate the decision criteria that maximized average perceived reward for each bird for different values of $w$. Finally, these decision criteria were used to calculate the percentage of correct and safe responses to each stimulus for each bird in both test phases. Thus, the model took the values of $d^{\prime}$ as input and tried to predict the percentages of sparse, dense, and safe responses by varying the relative perceived rewards of different types of correct responses. The value of $w$ that best fit the data was 1.45.

The predictions for the best-fitting model are shown in Table 1 and Figure 3. The correlations between the observed and predicted criteria, percentage of correct responses, and percentage of safe key responses, were $.98, .93$, and .97 , respectively. There is some indication that the birds adopted decision criteria that were slightly more conservative than the ones predicted by the model: They tended to select the dense response less frequently than the model predicted. However, the deviations from the predictions are small ( 0.2 standard deviations, on average) and do not appear to vary systematically across conditions. Overall, the model fits the data exceptionally well and provides support for the view that (1) the relative perceived rewards for matching and safe key responses were similar across birds and test phases, and (2) the birds adjusted their decision criteria to maximize the average perceived reward.

If perceived reward was proportional to the number of food pellets, $w$ would have been two. The fact that the best-fitting value of $w$ was 1.45 suggests that the perceived 

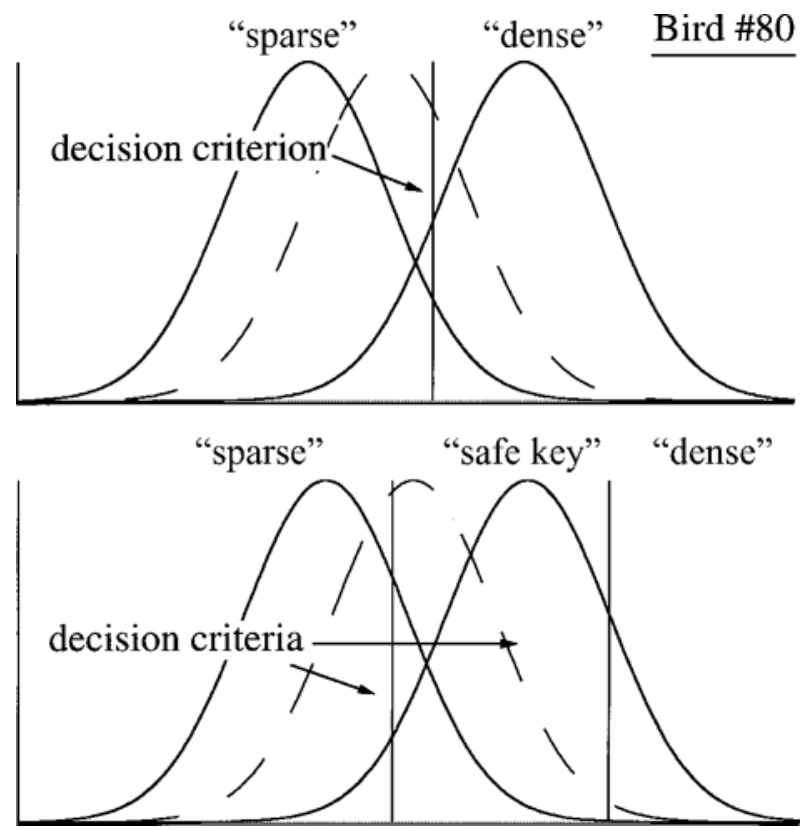

Figure 2. An illustration of the decision space used to model responses on matching and combined trials. The signal detection model assumed that the internal responses to sparse, variable (dotted distribution), and dense stimuli were random Gaussian variables with different means and identical variances. On matching trials, the decision axis is divided into two regions by one criterion (top). Internal responses falling below and above the criterion are classified as sparse and dense, respectively. On combined trials, the decision axis is divided into three regions by two decision criteria. Internal responses that fall below both criteria are classified as sparse, responses that fall above both criteria are classified as dense, and responses falling in between the criteria lead to the selection of the safe key. The distances between the sparse, variable, and dense distributions are calculated from Bird 80's data. Note that the distributions are closer together in the combined trials, corresponding to the finding that $d^{\prime}$ was lower on combined trials.

value of a correct matching response was attenuated relative to the perceived value of a safe key response. This attenuation might reflect the fact that the perceived reward of food simply grows as a compressive, nonlinear function of the number of pellets (see Bateson \& Kacelnik, 1995; Hamm \& Shettleworth, 1987). Another possibility is that the attenuation reflects the greater variability associated with selecting the sparse or dense keys. Selecting sparse or dense keys resulted in correct and incorrect responses, and therefore, reward varied across trials, but selecting the safe key always resulted in a reward of $x$ pellets. Thus, the apparently undue preference for the safe key may be seen as an example of risk aversion-that is, preference for a constant immediate reward over a mixture of six and zero pellets that is dependent on the proportion of correct matching responses. Animal responses to such variable outcomes can be explained without reference to sensitivity to uncertainty per se (Bateson \& Kacelnik, 1997).
Figure 1 shows that the birds responded very accurately to sparse stimuli. Even on the combined trials, percentage correct is greater than $95 \%$ in all the birds. If the birds were so accurate, why did they ever bother with the safe key on combined trials when a sparse stimulus was presented? It is important to note that the model assumes that the only basis for a response is a continuous internal response corresponding to stimulus density. According to this view, the birds do not perceive a sparse stimulus and then select the safe key. Instead, the birds are assumed to select the safe key when the stimulus (sparse, dense, or variable) evokes an internal response that falls within a certain range. Sparse stimuli occasionally produce internal responses in that range, but more frequently, those responses are caused by variable stimuli (see Figure 2). Using the safe key on sparse trials will reduce the average reward to those stimuli, but using the safe key increases the average reward to variable stimuli, because a lot of matching errors are made to that stimulus. Variable stimuli occur more frequently than sparse stimuli, so using the safe key increases overall reward.

All the birds exhibited lower $d^{\prime}$ on combined trials than on matching trials (Table 1). This corresponds to the fact that matching responses were not more accurate when the safe key was provided. It is surprising, because if the safe key is chosen selectively on difficult trials, in the region of overlap between sparse and dense density impressions and if $d^{\prime}$ does not change, the trials in which the safe key is rejected should be easiest to classify. Hampton's (2001) data on monkey metamemory fit this description. Two possible reasons for the pigeons' poorer performance on trials with than on trials without the safe key are that it is more difficult to maintain two stable criteria than one and that the birds were less familiar with the response display on combined trials, because they had been trained extensively on matching and with the safe key alone before combined trials were introduced (see note 1 ). In addition, it is possible that there are species differences in the performance of metacognitive tasks.

Like us, Smith and his colleagues (Shields et al., 1997; Smith et al., 1995; Smith et al., 1997) compared the behavior of their subjects with optimal behavior derived from signal detection theory. However, unlike in our experiment, their near-threshold stimuli changed dynamically throughout many of their experiments. The more accurately such stimuli were classified, the closer to threshold they became. Also unlike the case in our experiment, choosing the uncertain response (analogous to our safe key) gave no immediate reward but, rather, access to an easy rewarded trial after a delay that increased the more often the uncertain response was used. The maximum total rewards per session in Smith et al.'s (1995; Smith et al., 1997) experiments could be attained by making occasional errors with easy stimuli near the extremes of the stimulus continuum and not escaping too often. Instead of doing this, however, both humans and nonhumans generally drove themselves into a situation in which the variable stimulus 
Table 1

Observed and Optimal Decision Criteria and Values of $d^{\prime}$ on Matching and Combined Trials in Both Phases of the Experiment

\begin{tabular}{|c|c|c|c|c|c|c|}
\hline Bird & $d^{\prime}(\mathrm{S}$ vs. V) & $d^{\prime}$ (S vs. D) & $\begin{array}{c}\text { Observed } \\
\text { Criterion* }\end{array}$ & $\begin{array}{l}\text { Optimal } \\
\text { Criterion }\end{array}$ & & \\
\hline \multicolumn{7}{|c|}{ Matching Trials-Test } \\
\hline 57 & 2.6 & 3.1 & 2.3 & 2.1 & & \\
\hline 78 & 1.9 & 3.5 & 2.3 & 2.4 & & \\
\hline 80 & 1.0 & 2.7 & 1.6 & 1.7 & & \\
\hline \multicolumn{7}{|c|}{ Matching Trials-Transfer } \\
\hline 57 & 1.9 & 2.6 & 1.9 & 1.8 & & \\
\hline 78 & 1.7 & 3.5 & 2.3 & 2.4 & & \\
\hline \multirow[t]{3}{*}{80} & 1.9 & 3.2 & 1.9 & 2.3 & & \\
\hline & & & $\begin{array}{c}\text { Observed } \\
\text { Criterion } 1\end{array}$ & $\begin{array}{l}\text { Observed } \\
\text { Criterion } 2\end{array}$ & $\begin{array}{l}\text { Predicted } \\
\text { Criterion } 1\end{array}$ & $\begin{array}{c}\text { Predicted } \\
\text { Criterion } 2\end{array}$ \\
\hline & \multicolumn{6}{|c|}{ Combined Trials-Test } \\
\hline 57 & 1.0 & 1.5 & -0.2 & 2.0 & -0.1 & 2.1 \\
\hline 78 & 1.3 & 2.3 & 1.0 & 2.4 & 0.7 & 2.1 \\
\hline 80 & 1.1 & 2.5 & 0.8 & 2.4 & 0.8 & 2.0 \\
\hline \multicolumn{7}{|c|}{ Combined Trials-Transfer } \\
\hline 57 & 1.0 & 1.5 & -0.1 & 2.1 & -0.1 & 2.1 \\
\hline 78 & 1.2 & 2.8 & 1.6 & 2.3 & 1.1 & 2.2 \\
\hline 80 & 0.7 & 2.2 & 0.6 & 1.9 & 0.6 & 1.8 \\
\hline
\end{tabular}

Note-Criteria are expressed as the number of standard deviations away from the mean of the $\mathrm{S}$ distribution. S, sparse; V, variable; $\mathrm{D}$, dense.

was very close to threshold and, thereby, obtained fewer rewards per session than the optimum. They were evidently expressing the well-documented (see Shettleworth, 1998) preference for immediate reward (i.e., from a correct response on a relatively easy trial) over maximizing reward across all trials.

The dolphin studied by Smith and colleagues (1995) showed behavior similar to vicarious trial and error on trials with near-threshold stimuli. By analogy, on trials without the safe key, our pigeons might be expected to take longer to choose between sparse and dense with difficult $\mathrm{RV}$ and GV samples than with dense or sparse samples. To test this hypothesis, we computed the median latency to make a choice after pecking the sample for each block of sessions and compared the overall means of this measure across blocks for all five samples, using an analysis of variance. In neither phase were there any significant differences in latency as a function of the type of sample or of the choice made, not surprisingly given the overall pattern of choice data. If anything, latencies were slightly longer with the sparse samples, the ones easiest to classify correctly. There were also no differences in latency to peck the various samples.

By Sessions 5-16 of the transfer phase (Figure 1), the birds had transferred both matching and use of the safe key to the novel RV stimulus. Table 2 shows transfer to RV in more detail, as performance in the first and second foursession blocks of the transfer phase. In the first block, the birds classified RV only at chance or below. One might suppose that if the birds "knew they did not know" what the RV stimulus was, they would choose the safe key especially often at first but would do so less as accuracy improved. However, although all the birds' proportions correct with
RV increased considerably in the second block, choices of the safe key changed very little. Although limited, these data do not suggest that the pigeons were pecking the safe key when they experienced a state of uncertainty.

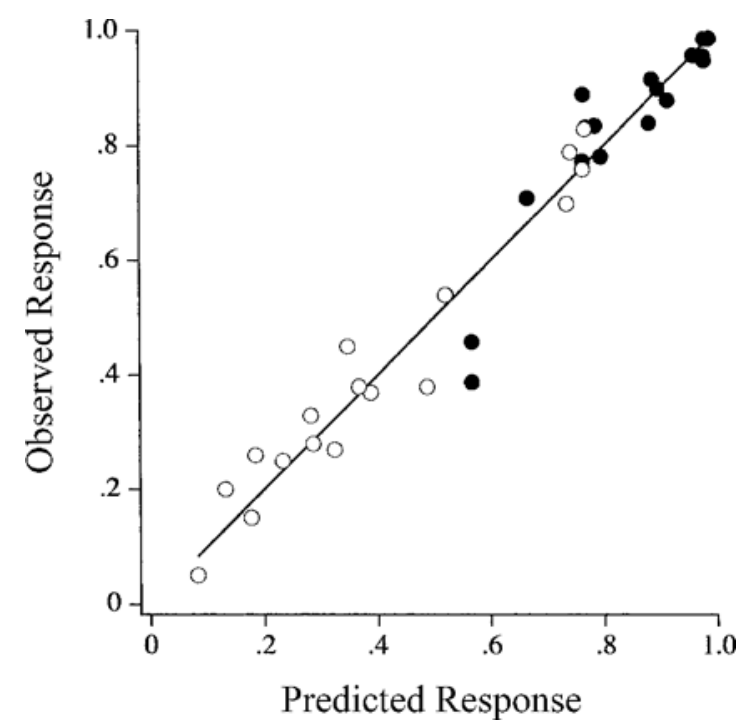

Figure 3. Graphical comparisons of the data and the predictions of the signal detection model. The observed proportions correct (filled symbols) and proportions of safe key responses (open symbols) for the primary stimuli in training and transfer phases (i.e., green ones in training, red in transfer) are plotted against the predictions of the model. Each point represents the proportion correct (or safe key) measured for one stimulus in a single bird. The correlations between the observed and the predicted proportions of correct-matching and safe key responses were .93 and .97 , respectively. 
Table 2

Proportions of Correct Classifications of the Red Variable (RV) Stimulus and Choices of the Safe Key on Combined Trials When RV Was the Sample in the First Two Blocks of Transfer

\begin{tabular}{ccccccccc}
\hline & \multicolumn{2}{c}{$\begin{array}{c}\text { Matching } \\
\text { Percentage Correct }\end{array}$} & & \multicolumn{2}{c}{$\begin{array}{c}\text { Combined } \\
\text { Percentage Correct }\end{array}$} & & \multicolumn{2}{c}{$\begin{array}{c}\text { Percentage for } \\
\text { Safe Key }\end{array}$} \\
\cline { 2 - 3 } Bird & Block 1 & Block 2 & & Block 1 & Block 2 & & Block 1 & Block 2 \\
\hline 57 & .38 & .46 & & .18 & .36 & & .82 & .85 \\
78 & .52 & .75 & & .47 & .79 & & .31 & .26 \\
80 & .42 & .81 & & .45 & .62 & & .6 & .53 \\
$M$ & .44 & .67 & & .37 & .59 & & .58 & .55 \\
\hline
\end{tabular}

From the pigeons' point of view, the task might have been a luminance discrimination, with color irrelevant. For a fixed color, the luminance of our stimuli was proportional to pixel density, and so it is conceivable that GS, $\mathrm{GV}$, and GD stimuli were discriminated on the basis of luminance. To evaluate this hypothesis, we measured spectral distribution functions (i.e., radiance in 4-nm bands from 380 to $720 \mathrm{~nm}$ ) for the green and the red phosphors of our stimulus display. Next, pigeon photopic spectral sensitivity functions (P. M. Blough, Riggs, \& Schafer, 1972) were used to convert the radiometric measures to photometric units (i.e., luminance). By our calculations, the red phosphor produced a luminance that was 0.4 times that of the green phosphor. This large difference means that the average luminance of the RD stimulus was only $47 \%$ of the GV luminance. If pigeons responded on the basis of luminance alone, we would expect the RD stimulus to be classified incorrectly as sparse. The fact that pigeons classified RD items correctly as dense during training and transfer (see Figure 1) makes it unlikely that the responses were based solely on average luminance.

In summary, the pigeons in our experiment performed similarly to the dolphin, monkeys, pigeons, and people in previous nonverbal tests of metacognition, in that they often chose an alternative to performing a cognitive task when that task was most difficult. In that respect, choice of the safe key was functionally similar to a person's report of uncertainty. However, in other respects, the birds failed the test of functional similarity. They did not behave as if they were sensitive, on a trial-by-trial basis, to whether or not they could classify a stimulus correctly, in that they did not perform any better when choosing to complete the primary discrimination task than when forced to. This contrasts with the behavior of pigeons in a comparable test of metamemory (Inman \& Shettleworth, 1999, Experiment 1) and that of monkeys in more stringent tests (Hampton, 2001; see also Smith et al., 1998). In addition, the birds did not choose the safe key especially often when a novel near-threshold stimulus was still unknown and they might have been expected to be uncertain about how to classify it. The data were well described by a signal detection model that assumed that the birds were maximizing perceived reward in a consistent way across all the experimental conditions. The use of such a model need not imply that the birds were explicitly setting criteria; in any case, alternative models without such criteria (e.g., White \& Wixted, 1999) may do equally well.
There is increasing interest in behavioral tests for nonhuman subjects that are designed to look for behavior having close functional similarities to verbal behavior in tests of conscious processes in adult humans. Besides illuminating the evolution of higher cognitive processes, such tests can underpin investigations of their neural basis (Morris, 2001). In addition to metamemory (Hampton, 2001) and perceptual uncertainty, examples include episodic memory (Clayton, Yu, \& Dickinson, 2001; Griffiths, Dickinson, \& Clayton, 1999), visual awareness (Cowey \& Stoerig, 1997), and theory of mind (Povinelli \& Eddy, 1996). In the few previous studies of metacognition in nonhuman subjects, the most stringent tests have provided evidence for metamemory in monkeys (Hampton, 2001; Smith et al., 1998), but not in pigeons (Inman \& Shettleworth, 1999, Experiment 2). Other studies have provided evidence consistent with monkeys' and a dolphin's ability to respond to perceptual uncertainty in the way people do (Shields et al., 1997; Smith et al., 1995; Smith et al., 1997). Here, we employed stimuli very similar to those used in one of the latter studies, although our method differed in other respects. As with metamemory, it appears that pigeons behave differently from the mammals that have been tested. Although they behaved adaptively, in that they maximized perceived reward, the pigeons apparently did so without relying on a process having the functional properties of conscious uncertainty.

\section{REFERENCES}

BAteson, M., \& KACELniK, A. (1995). Accuracy of memory for amount in the foraging starling, Sturnus vulgaris. Animal Behaviour, 50, 431443.

BAteson, M., \& KACELnIK, A. (1997). Starlings' preferences for predictable and unpredictable delays to food. Animal Behaviour, 53, 1129-1142.

Blough, D. S. (1967). Stimulus generalization as signal detection in pigeons. Science, 158, 940-941.

Blough, P. M., Riggs, L. A., \& Schafer, K. L. (1972). Photopic spectral sensitivity determined electroretinographically for the pigeon eye. Vision Research, 12, 477-486.

Boneau, C. A., \& Cole, J. L. (1967). Decision theory, the pigeon, and the psychophysical function. Psychological Review, 74, 123-135.

Clark, R. E., \& SQuire, L. R. (1998). Classical conditioning and brain systems: The role of awareness. Science, 280, 77-81.

Clayton, N. S., Yu, K. S., \& Dickinson, A. (2001). Scrub jays (Aphelocoma coerulescens) form integrated memories of the multiple features of caching episodes. Journal of Experimental Psychology: Animal Behavior Processes, 27, 17-29.

Commons, M. L., Nevin, J. A., \& Davison, M. C. (Eds.) (1991). Signaldetection: Mechanisms, models, and applications. Hillsdale, NJ: Erlbaum. 
Cowey, A., \& Stoerig, P. (1997). Visual detection in monkeys with blindsight. Neuropsychologia, 35, 929-939.

GrifFIN, D. R. (2001). Animals know more than we used to think. Proceedings of the National Academy of Sciences, 98, 4833-4834.

Griffiths, D., Dickinson, A., \& Clayton, N. (1999). Episodic memory: What can animals remember about their past? Trends in Cognitive Sciences, 3, 74-80.

HAmm, S. L., \& Shettleworth, S. J. (1987). Risk aversion in pigeons. Journal of Experimental Psychology: Animal Behavior Processes, 13, 376-383.

Hampton, R. R. (2001). Rhesus monkeys know when they remember. Proceedings of the National Academy of Sciences, 98, 5359-5362.

InMAN, A., \& Shettleworth, S. J. (1999). Detecting metamemory in nonverbal subjects: A test with pigeons. Journal of Experimental Psychology: Animal Behavior Processes, 25, 389-395.

Kacelnik, A., \& Bateson, M. (1996). Risky theories: The effects of variance on foraging decisions. American Zoologist, 36, 402-434.

Macmillan, N. A., \& Creelman, C. G. (1991). Detection theory: A user's guide. Cambridge: Cambridge University Press.

MacphaIL, E. M. (1998). The evolution of consciousness. Oxford: Oxford University Press.

Marston, H. M. (1996). Analysis of cognitive function in animals, the value of SDT. Cognitive Brain Research, 3, 269-277.

Morris, R. G. M. (2001). Episodic-like memory in animals: Psychological criteria, neural mechanisms and the value of episodic-like tasks to investigate animal models of neurodegenerative disease. Philosophical Transactions of the Royal Society of London: Series B, 356, 1453-1465.

Nelson, T. O. (1996). Metacognition and consciousness. American Psychologist, 51, 102-116.

Povinelli, D. J., \& EDDY, T. J. (1996). What young chimpanzees know about seeing. Monographs of the Society for Research in Child Development, 61, 1-152.

Shettleworth, S. J. (1998). Cognition, evolution, and behavior. New York: Oxford University Press.

Shettleworth, S. J. (2001). Animal cognition and animal behaviour. Animal Behaviour, 61, 277-286.

Shields, W. E., Smith, J. D., \& Washburn, D. A. (1997). Uncertain responses by humans and rhesus monkeys (Macaca mulatta) in a psychophysical same-different task. Journal of Experimental Psychology: General, 126, 147-164.

Smith, J. D., Schull, J., Strote, J., McGee, K., Egnor, R., \& Erb, L. (1995). The uncertain response in the bottlenosed dolphin (Tursiops truncatus). Journal of Experimental Psychology: General, 124, 391-408.

Smith, J. D., Shields, W. E, Allendoerfer, K. R., \& Washburn, D. A. (1998). Memory monitoring by animals and humans. Journal of Experimental Psychology: General, 127, 227-250.
Smith, J. D., Shields, W. E., Schull, J., \& Washburn, D. A. (1997). The uncertain response in humans and animals. Cognition, 62, 75-97. White, K. G., \& WiXTED, J. T. (1999). Psychophysics of remembering. Journal of the Experimental Analysis of Behavior, 71, 91-113.

WiXTED, J. T. (1993). A signal detection analysis of memory for nonoccurrence in pigeons. Journal of Experimental Psychology: Animal Behavior Processes, 19, 400-411.

\section{NOTES}

1. The training leading up to final performance consisted of three phases. First, the birds were trained in 10-12 48-trial sessions to discriminate the two dense from the two sparse stimuli at a level of $90 \%$ accuracy over 4 consecutive sessions. Second, safe key trials were introduced while the same accuracy was maintained for 4 sessions of 48 matching and 48 safe key trials. Third, GV trials with $30 \%$ green pixels were introduced. A sparser green pixel density barely discriminable from the GD sample was determined for each bird by increasing the density on $\mathrm{GV}$ trials by $5 \%$ per session as long as percentage correct was at least $63 \%$ and performance on GS, RS, and RD was maintained at $90 \%$ correct. Failure to reach this criterion within 6 sessions at a given GV density concluded this stage and selected that value as the GV and RV density in the next stages. When performance fell below $58 \%$ correct for a particular GV density on 6 consecutive sessions, GV density was lowered by $2 \%$. This process continued until a GV density was found on which the pigeon maintained $58 \%-62 \%$ correct over 6 sessions. The density selected for all 3 birds was $70 \%$. Sessions at this stage consisted of 112 trials, with GD, GS, GV, RD, and RS samples intermixed in the ratio 6:2: 4:1:1 in both matching and safe key trials. This stage lasted between 20 and 33 sessions and included GV densities from $30 \%$ to $73 \%$. Neither $\mathrm{RV}$ samples nor combined trials occurred at this stage.Testing on combined trials began immediately after determination of the final GV value.

2 . When the perceived value is proportional to the number of food pellets, the strategy that maximizes the average reward is identical to the single-criterion strategy used on trials when the safe key was absent. To see why this is so, consider the performance of a bird that failed to encode any stimulus information and simply selected safe or dense responses at random: The average reward would be three pellets. A bird that is capable of extracting any information from the display would, in the long run, be correct on more than $50 \%$ of the trials, and so the average reward would be greater than three. Selecting the safe key always yields an average reward of three pellets, and therefore, using an optimal, single-criterion strategy will always yield an average reward that is equal to or greater than the reward produced by selecting the safe key.

(Manuscript received October 18, 2000; revision accepted for publication July 18, 2002.) 